\title{
The involvement of follistatin-like protein 1 in osteoarthritis by elevating NF-KB-mediated inflammatory cytokines and enhancing fibroblast like synoviocyte proliferation
}

\author{
Su Ni ${ }^{1,2+}$, Kaisong Miao ${ }^{1,2 \dagger}$, Xianju Zhou ${ }^{3}$, Nanwei Xu ${ }^{1}$, Chenkai Li ${ }^{1,2}$, Ruixia Zhu ${ }^{1}$, Rongbin Sun ${ }^{1}$ and Yuji Wang ${ }^{1,2^{*}}$
}

\begin{abstract}
Introduction: Our previous work has revealed that expression of follistatin-like protein 1 (FSTL1) is elevated in the synovial tissues from osteoarthritis (OA) patients. The aim of this study was to elucidate the underlying molecular mechanisms by which FSTL1 plays a role in the pathogenesis of OA.

Methods: Cultured fibroblast-like synoviocytes (FLSs) from synovial tissues of OA patients were stimulated with human recombinant FSTL1, and then the expression of inflammatory cytokines in FLS and their concentrations in the cell supernatants were measured by real-time polymerase chain reaction (PCR) and enzyme-linked immunosorbent assay (ELISA), respectively. Nuclear factor kappa B (NF-KB) activation was examined by western blot and chromatin immunoprecipitation (ChIP) assay at the p65 binding site. Finally, the proliferation of FLSs and the expression level of the proliferation-related tumor suppressors (p53 and p21) were determined by MTS assay kit and western blot in the presence or absence of FSTL1, respectively.

Results: FSTL1 remarkably promoted expression levels of several inflammatory cytokines (tumor necrosis factor alpha (TNF-a), interleukin-1 $\beta(I L-1 \beta)$ and interleukin-6 (IL-6)) in vitro. Western blot analysis showed that FSTL1 activated the inflammatory-related NF-KB signaling pathway, as validated by ChIP assay detecting p65-binding level on the cytokine promoter region. Moreover, FSTL1 promoted the proliferation of OA FLS by downregulating the expression of p53 and p21. Interestingly, the concentration of synovial fluid IL-6 was remarkably elevated in OA patients, and was correlated with synovial fluid and serum FSTL1 levels.
\end{abstract}

Conclusions: These findings show that FSTL1 functions as an important proinflammatory factor in the pathogenesis of OA by activating the canonical NF-KB pathway and enhancing synoviocytes proliferation, suggesting that FSTL1 may be a promising target for the treatment of $\mathrm{OA}$.

\section{Introduction}

Osteoarthritis (OA) is a rheumatic disorder with the highest prevalence among all arthritis. Knee OA causes to most of mobility disability in the elderly over 65 [1]. As the degradation of articular cartilage proceeds, OA patients suffer

\footnotetext{
* Correspondence: yujiwang@sohu.com

'Equal contributors

'Department of Orthopedics, The Affiliated Hospital of Nanjing Medical University, Changzhou No.2 People's Hospital, 29 Xinglong Alley, Changzhou 213003, China

'Laboratory of Clinical Orthopedics, The Affiliated Hospital of Nanjing Medical University, Changzhou No.2 People's Hospital, 29 Xinglong Alley, Changzhou 213003, China

Full list of author information is available at the end of the article
}

from a variety of symptoms including joint pain, tenderness, stiffness, locking, and sometimes an effusion. Importantly, the synovium and cartilage of the OA patients are often subject to extreme changes of microenvironment triggered largely by inflammatory cytokines and proteases in extracellular matrix (ECM), and thus leading to dysfunction of various types of cell in synovitis joints, especially fibroblast-like synoviocytes (FLSs) [2,3]. Inflammation of the synovium is frequently involved in the pathological process, even in the early stages of OA $[4,5]$. A growing body of evidence indicates the importance of activated FLS in OA pathogenesis. FLS promotes cartilage degradation through the secretion of inflammatory/catabolic mediators 
and other soluble factors [6-8]. Therefore, cultured FLS is a good in vitro model for OA pathogenesis and drug target discovery [6,9].

Among multiple pathways and mediators influencing the development and persistence of $\mathrm{OA}$, nuclear factorkappa $\mathrm{B}(\mathrm{NF}-\mathrm{kB})$ transcription factor plays a prominent role $[10,11]$. The NF-кB family consists of P50 (NF-кB1), P52 (NF-кB2), P65 (RelA), RelB and C-Rel. NF-кB protein, a heterodimer of p50 and p65/RelA, is usually sequestered by inhibitor of kappa $B$ alpha $\left(I_{\kappa} B \alpha\right)$ in the cytosol in the inactive state $[12,13], \mathrm{NF}-\mathrm{kB}$ can be activated by proinflammatory cytokines, excessive mechanical stress and ECM degradation enzymes. Once the NF- $\mathrm{kB}$ signaling cascade is activated, a complete degradation of IкB following phosphorylation by upstream ІкB kinases (IKKs) allows the translocation of the Rel/P50 homodimer to the nucleus and subsequently induces gene transcription $[14,15]$. Activated NF- $\mathrm{kB}$ boosts the expression of many inflammation-related cytokines and chemokines, such as interleukin 6 (IL-6), interleukin 8 (IL-8), adhesion molecules and matrix-degrading enzymes, and thus regulating inflammation, immune functions and cellular growth [16,17]. Therefore, NF-кB-activating kinases are one of the potential therapeutic OA targets [10].

Follistatin-like protein 1 (FSTL1), which was first identified as a transforming growth factor $\beta 1$ (TGF- $\beta 1$ )-inducible protein, is a secreted extracellular glycoprotein containing a follistatin-like and extracellular calcium-binding domain [18]. It has been reported that FSTL1 is involved in diverse biological processes including cell proliferation and differentiation, wound healing, inflammation, skeletal muscle growth and fibrosis [19-21]. Previous studies revealed a substantial connection between the FSTL1 level and severity of rheumatoid arthritis [22,23]. Moreover, our past work showed that FSTL1 acts as a serum biochemical marker of OA and reflects the severity of joint damage in patients [24].

However, the underlying mechanisms by which FSTL1 plays a role in the pathogenesis of OA are poorly understood. In view of the crucial role of FLS in OA progression $[2,11]$, this study was aimed at investigating the signal transduction of FSTL1 in cultured OA FLS, and revealing the potential roles of FSTL1 in OA pathogenesis.

\section{Material and methods}

\section{Synovial fluid and serum samples}

Serum and synovial fluid (SF) samples were obtained from 58 OA patients by synovectomy or joint replacement surgery. OA serum and SF samples were obtained from patients diagnosed with knee OA (of KellgrenLawrence (KL) score 1 to 4 [25]) according to the 1985 criteria of the American Rheumatism Association [26]. The clinical and demographic characteristics of OA patients and their controls are shown in Table 1. The SF
Table 1 Characteristics of the subjects investigated ${ }^{a}$

\begin{tabular}{|c|c|c|}
\hline & $\mathrm{HC}$ & $O A$ \\
\hline Number & 30 & 58 \\
\hline Age (years) $^{b}$ & $60(55-64)$ & $66(60-75)$ \\
\hline $\operatorname{Sex}(M / F)$ & $16 / 14$ & $25 / 33$ \\
\hline Disease duration (years) ${ }^{\mathbf{b}}$ & - & $1.0(0.5-3.0)$ \\
\hline KL grade (0-4) & - & $0 / 11 / 14 / 23 / 10$ \\
\hline
\end{tabular}

ancluded all subjects for whom serum samples were available, and all the SF samples and primary cell lines from OA subjects were available; ${ }^{\text {b expressed as }}$ the median (25th to 75th percentile). HC, healthy control individual; OA, osteoarthritis; KL, Kellgren-Lawrence; SF, synovial fluid.

samples obtained were centrifuged for $5 \mathrm{~min}$ at 10,000 rpm to remove the obvious contamination with blood or debris. A total of 30 serum samples of apparently healthy individuals diagnosed without alcohol abuse or chronic drug or other chronic disease or acute illness were included as healthy controls (HCs).This study was reviewed and approved by the ethics committee of Changzhou No.2 People's Hospital. Informed consent was received from all patients and controls.

\section{Isolation and culture of human FLSs}

Synovial tissue specimens were obtained from OA patients by synovectomy or joint replacement surgery. Tissues were carefully minced and digested with $1 \mathrm{mg} / \mathrm{ml}$ collagenase I (Sigma-Aldrich, St. Louis, MO, USA) in serum-free Dulbecco's modified Eagle's medium (DMEM) (Gibco BRL, Grand Island, NY, USA) for 4 to 6 hrs at $37^{\circ} \mathrm{C}$, filtered through a $70-\mu \mathrm{m}$ cell strainer (BD, Durham, NC, USA), extensively washed with DMEM blank and finally cultured in DMEM supplemented with $10 \%$ fetal calf serum (Gibco BRL, Grand Island, NY, USA), 100 U penicillin, and $100 \mu \mathrm{g} / \mathrm{ml}$ streptomycin in a standard cell culture chamber containing $5 \% \mathrm{CO}_{2}$. The nonadherent cells were removed next day. The adherent cells were cultured up to $90 \%$ confluence and then split in $1 / 3$ ratio up to passage 3-6 (consisting of a homogeneous population). The cultured FLSs at these passages were used for subsequent experiments.

\section{Reagents and stimulation assays}

Each FLS line from an individual OA patient was used for each experiment, which was repeated two times with different OA FLS lines. Cultured FLSs were grown in $100-\mathrm{mm}$ cell culture dishes ( 8 to $10 \times 10^{5}$ cells/dish) for western blot or in 6-well plates ( 1 to $1.5 \times 10^{5}$ cells/well) for mRNA extraction. Cultures were stimulated with human recombinant FSTL1 (Adipo Bioscience, Santa Clara, CA, USA), which was dissolved in phosphate-buffered saline (PBS) solution, or vehicle (PBS solution). Stimulation was applied for 24 or $48 \mathrm{hrs}$ for mRNA extraction, or for 30 min for NF- $\mathrm{kB}$ activation, or 48 hrs for supernatant collection. BAY 11-7082 (Selleckchem, Houston, 
TX, USA), a specific inhibitor of NF-kB signaling pathway, was dissolved in dimethyl sulphoxide (DMSO) solution to the concentration of $5 \mathrm{mM}$, and added to the cultures at ratio of 1:1,000 (final concentration was $5 \mu \mathrm{M})$ [27] for $1 \mathrm{hr}$ pretreatment.

\section{Cell proliferation}

Cell proliferation assay was performed using a novel tetrazolium compound (3-(4,5-dimethylthiazol-2-yl)-5-(3carboxymethoxyphenyl)-2-(4-sulfophenyl)-2H-tetrazolium, inner salt; MTS) and an electron coupling reagent (phenazine ethosulfate; PES)-based CellTiter $96^{\text {ma }}$ AQueous One Solution Cell Proliferation (MTS) assay (Promega, Madison, WI, USA). OA FLSs were seeded at about 2,000 cells per well in 96-well plates in triplicate for 7 days under regular growth conditions (DMEM with 10\% fetal bovine serum (FBS)), and then MTS cell viability assay was performed daily according to the manufacturer's instructions. Twelve hours after seeding, FSTL1 was added daily in the medium, which was changed every two days.

\section{Real-time polymerase chain reaction (PCR)}

Total RNA was extracted from cultured FLSs using NucleoSpin RNA Kit (MN, Düren, Germany) according to the manufacturer's instructions. One $\mu \mathrm{g}$ total RNA was reverse-transcribed using the High-Capacity cDNA Reverse Transcription Kit (Applied Biosystems, Foster City, CA, USA) according to the manufacturer's instructions. A quantitative real-time PCR assay was carried out using SYBR $^{\mathrm{Tw}}$ Select Master Mix (Applied Biosystems, Austin, TX, USA) in a Bio-Rad iQ5 (Bio-Rad Laboratories, Hercules, CA, USA). Primer sequences for amplifying human cytokines cDNA and internal control glyceraldehyde 3-phosphate dehydrogenase (GAPDH) were as follows: IL$1 \beta, 5^{\prime}$ - AAGCTGAGGAAGATGCTG -3' (forward) and 5' - ATCTACACTCTCCAGCTG -3' (reverse); IL-6, 5'GAACTCCTTCTCCACAAGCGCCTT -3' (forward) and 5'- GACCAGTGATGATTTTCACCAGG -3' (reverse); TNF-a, 5' - CCAGGCAGTCAGATCATCTTCTC -3' (forward) and 5' - AGCTGGTTATCTCTCAGCTCCAC -3' (reverse); GAPDH, 5'-AGGGCTGCTTTTAACTCTGGT3' (forward) and 5'-CCCCACTTGATTTTGGAGGGA-3' (reverse). The comparative threshold cycle method was used for relative quantification of mRNA.

\section{Western blot}

Cultured FLSs were lysed in RIPA and boiled. Sodium dodecyl sulfate (SDS)-polyacrylamide gel electrophoresis was conducted on $10 \%$ polyacrylamide gel and transferred to polyvinylidene fluoride (PVDF) membrane. Rabbit anti-human p65 polyclonal antibody, rabbit anti-human phosphate p-65 polyclonal antibody, rabbit anti-human IкB polyclonal antibody, mouse antihuman phosphate IKB monoclonal antibody, and rabbit anti-human p50 polyclonal antibody (all these antibodies were purchased from Cell Signaling Technology, Danvers, MA, USA) were used to detect the corresponding protein in the NF- $\mathrm{KB}$ signaling pathway. Rabbit anti-human actin polyclonal antibody was used to detect actin signal as an internal loading control and relative expression levels were quantified by running the Quantity One software (Bio-Rad Laboratories, Hercules, CA, USA).

\section{Chromatin immunoprecipitation (ChIP)}

Cultured FLSs were subjected to $5 \mu \mathrm{g} / \mathrm{ml}$ FSTL1 or vehicle (PBS solution) for 12 hrs. A total of $10^{7}$ cells were fixed and cross-linked in fresh $1 \%$ formaldehyde for 10 min and then quenched with $2.5 \mathrm{M}$ glycine for $5 \mathrm{~min}$ at room temperature. Cells were then harvested and suspended in lysis buffer. Simple ChIP ${ }^{\mathrm{mm}}$ Enzymatic Chromatin IP Kit (Cell Signaling Technology, Danvers, MA, USA) was used according to the manufacturer's instructions. Chromatin was digested with micrococcal nuclease, sheared by sonication and then lysates were clarified by centrifugation at $10,000 \mathrm{rpm}$ for $10 \mathrm{~min}$ at $4^{\circ} \mathrm{C}$. The supernatant was incubated with ChIP-grade rabbit antihuman p65 polyclonal antibody (Cell Signaling Technology, Danvers, MA, USA) or normal immunoglobulin G (IgG) overnight at $4^{\circ} \mathrm{C}$ with rotation. After being pulled down with protein $\mathrm{G}$ agarose beads, the target proteinDNA complexes were sequentially washed several times and reverse cross-linked to elute DNA for the subsequent experiment. DNA was purified and real-time PCR was performed to evaluate the ChIP-enriched DNA. Primer sequences for detecting p65-binding tumor necrosis factor alpha (TNF- $\alpha$ ) promoter region were 5' - ATATGGCCA CACACTGGGGC -3' (forward) and 5'- GGGCTTGG TGGCAGGCTTGA -3' (reverse). IL-6 promoter region were $5^{\prime}$ - ACCCTCACCCTCCAACAAAG-3' (forward) and 5' - GCCTCAGACATCTCCAGTCC -3' (reverse).

\section{Enzyme-linked immunosorbent assay (ELISA)}

TNF- $\alpha$, interleukin $1 \beta$ (IL-1 $\beta$ ), and IL- 6 levels in cell supernatants were measured using a standard quantitative sandwich ELISA (eBioscience, Vienna, Austria). All analyses and calibrations were performed in duplicate. Optical densities were determined using an absorbance microplate reader (Elx808 ${ }^{\mathrm{mi}}$ Bio-Tek Instruments, Winooski, VT, USA) at $450 \mathrm{~nm}$. Gen5 Data Analysis software (Bio-Tek Instruments, Winooski, VT, USA) was used to analyze all data and depict the standard curve.

\section{Statistical analysis}

Statistical analysis was performed using Prism (GraphPad Software, San Diego, CA, USA) and SPSS 17.0 software (SPSS Inc., Chicago, IL, USA). $X^{2}$ test was used for sex difference between OA patients and the controls; 
Spearman's rank correlation test was used to evaluate the associations between serum/SF FSTL1 level and SF IL-6 level; the significance of other differences was evaluated using Student's $t$ test. $P$ values less than 0.05 were considered significant.

\section{Results}

FSTL1 induces expression and secretion of inflammatory cytokines from OA FLS

Previous studies showed that FSTL1 expression correlates with severity of arthritis both in OA patients and mice models [22,24]. And a connection between cytokine/chemokine level and FSTL1 expression has been established in mice arthritis models [22]. To further examine FSTL1 functions, we used primary FLSs from OA patients' synovial tissues as model cells. First, we incubated FLSs with the two different FSTL1 concentrations $(1 \mu \mathrm{g} / \mathrm{ml}$ and $5 \mu \mathrm{g} / \mathrm{ml})$ for $24 \mathrm{hrs}$, we observed that FSTL1 remarkably increased the level of TNF- $\alpha$, IL-1 $\beta$ and IL- 6 mRNA in the FLSs in a dose-dependent way
(Figure 1A). Surprisingly, the increment of IL-6 mRNA was extremely prominent (more than 200-fold). Moreover, $10 \mu \mathrm{g} / \mathrm{ml}$ FSTL1 treatment did not result in a further increase in these mRNA levels compared to the treatment with $5 \mu \mathrm{g} / \mathrm{ml}$ FSTL1 (data not shown). Next, FLSs were incubated with $5 \mu \mathrm{g} / \mathrm{ml} \mathrm{FSTL1} \mathrm{for} \mathrm{two} \mathrm{differ-}$ ent times (24 and $48 \mathrm{hrs).The} \mathrm{expression} \mathrm{level} \mathrm{of} \mathrm{the}$ three inflammatory cytokines increased with the incubation time (Figure 1B). Consistently, the three secreted protein levels in the supernatant were markedly elevated following $48 \mathrm{hrs}$ of treatment with FSTL1 at $5 \mu \mathrm{g} / \mathrm{ml}$ (Figure 1C-E). Especially, elevation of released IL-6 was more than 500-fold. Together, these data further support our previous work that FSTL1 reflects the severity of OA by promoting the release of inflammatory cytokines in articular cavity

\section{FSTL1 activates NF-KB signaling in OA FLS}

NF- $\mathrm{BB}$ signaling is a canonical pathway that participates in immunity and inflammation $[28,29]$. Thus we wanted

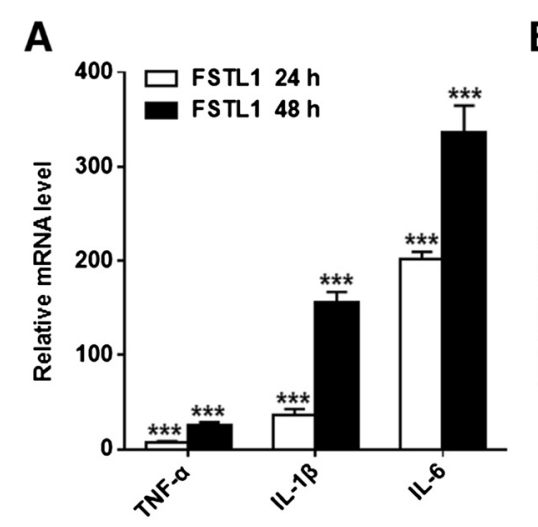

B


Figure 1 FSTL1 increases expression and excretion of inflammatory cytokines in cultured OA FLS. (A) Cultured FLSs were treated with FSTL1 (dissolved in PBS solution) in different concentration $(1 \mu \mathrm{g} / \mathrm{ml}$ or $5 \mu \mathrm{g} / \mathrm{ml}$ ) for $24 \mathrm{hrs}$. (B) Cultured FLSs were stimulated with $5 \mu \mathrm{g} / \mathrm{ml}$ FSTL1 for two different durations (24 or 48 hrs). In (A) and (B), total RNA was extracted and reverse transcribed, and the relative levels of TNF-a, IL-1 $\beta$ and IL-6 mRNA were analyzed by real-time PCR. The relative level of mRNA expression was normalized to the level of housekeeping gene GAPDH expression, and presented as fold changes relative to the control (the level of the control was set as 1). (C-E) Cell supernatant was collected from the cultures treated with $5 \mu \mathrm{g} / \mathrm{ml}$ FSTL1 for 48 hrs. TNF- - , IL-1 $\beta$ and IL- 6 concentrations were measured by ELISA. The above results are presented as mean \pm (standard deviation) SD of three independent experiments (each FLS line from an individual OA patient was used for each experiment). ${ }^{*} P<0.05,{ }^{* *} P<0.01,{ }^{* * *} P<0.001$, compared to the control (the vehicle-treated group), Student $t$ test. ELISA, enzyme-linked immunosorbent assay; FLS, fibroblast-like synoviocyte; FSTL1, follistatin-like protein 1; IL-1 $\beta$, interleukin-1 $\beta$; IL-6, interleukin-6; OA, osteoarthritis; PBS, phosphate-buffered saline; TNF-a, tumor necrosis factor alpha. 
to know whether FSTL1 exerts its inflammatory effects by activation of NF-kB signaling. By western blot analysis, we found that the level of phosphorylated p65

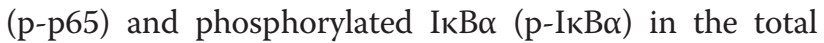
cell lysate were significantly increased following $5 \mu \mathrm{g} / \mathrm{ml}$ FSTL1 stimulation for $30 \mathrm{~min}$ (Figure 2A). In contrast, the expression of NF- $\mathrm{kB}$ subunit p65 and its intracellular inhibitor IкB $\alpha$ did not show significant changes (Figure 2A). Next, we separated the contents of the nucleus and carried out western blot analysis. A marked increase of p-p65, but not p50/105, was observed in the nucleus (Figure 2B). To further link NF- $\mathrm{kB}$ activation to downstream transcriptional activity, we performed ChIP assay. As compared to the control, FSTL1 promoted more p65 subunits to bind to the TNF- $\alpha$ and IL- 6 promoter region, suggesting transcription activation of TNF- $\alpha$ and IL-6 (Figure 2C-D). And the enrichment folds were approximately 1.5 and 8 respectively, in line with the pattern of the mRNA increments. Next, we utilized BAY 11-7082 (5 $\mu \mathrm{M})$, an effective inhibitor of IкB $\alpha$ phosphorylation, to specifically abrogate NF- $\mathrm{BB}$ DNA binding, and eventually downregulate the NF-kB-inducible cytokine levels. Compared to the control group, BAY 11-7082 significantly attenuated the FSTL1triggered increase in the expression of inflammatory cytokines (Figure 3A-D). And the inhibitor itself did not affect the baseline level of these cytokines. Taken together,
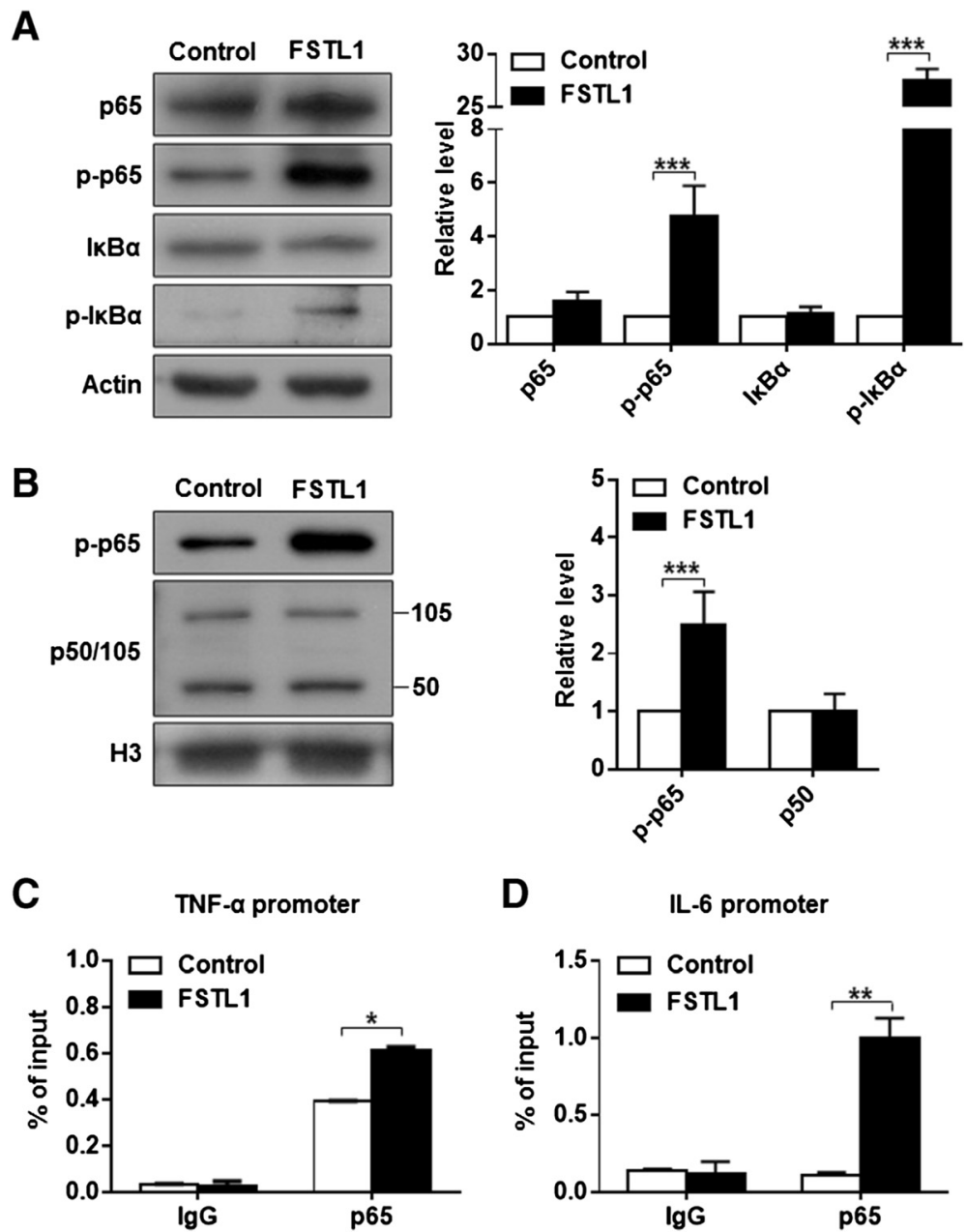

Figure 2 FSTL1 activates the NF-KB pathway in FLS. (A) Following treatment, the levels of key components of NF-KB in total cell lysates were determined by western blot analysis. (B) Nuclear extracts were isolated to further confirm the activation of NF-KB pathway. In (A) and (B), FLSs were treated with FSTL1 $(5 \mu \mathrm{g} / \mathrm{ml})$ or vehicle (PBS solution) for $30 \mathrm{~min}$. Representative western blot (Left) and quantification data (Right) are shown for the corresponding groups. The relative protein levels were normalized to the level of the internal control, actin or histone 3 (H3), and presented as fold changes relative to the control group (the level of the control group was set as 1). (C) and (D) FLSs were treated with or without FSTL1 for 12 hrs, and then ChIP assay targeting the p65 binding site on TNF-a (C) and IL-6 promoter (D) was performed. Data were shown as the percentage of input. The above results are presented as mean \pm (standard deviation) SD of three independent experiments (each FLS cell line from an individual patient was used for each experiment). ${ }^{*} P<0.05,{ }^{* *} P<0.01,{ }^{* * * *} P<0.001$, compared to the vehicle-treated group, Student $t$ test. ChIP, chromatin immunoprecipitation; FLS, fibroblast-like synoviocyte; FSTL1, follistatin-like protein 1; IL-6, interleukin-6; NF-KB, nuclear factor kappa B; PBS, phosphate-buffered saline; TNF-a, tumor necrosis factor alpha. 

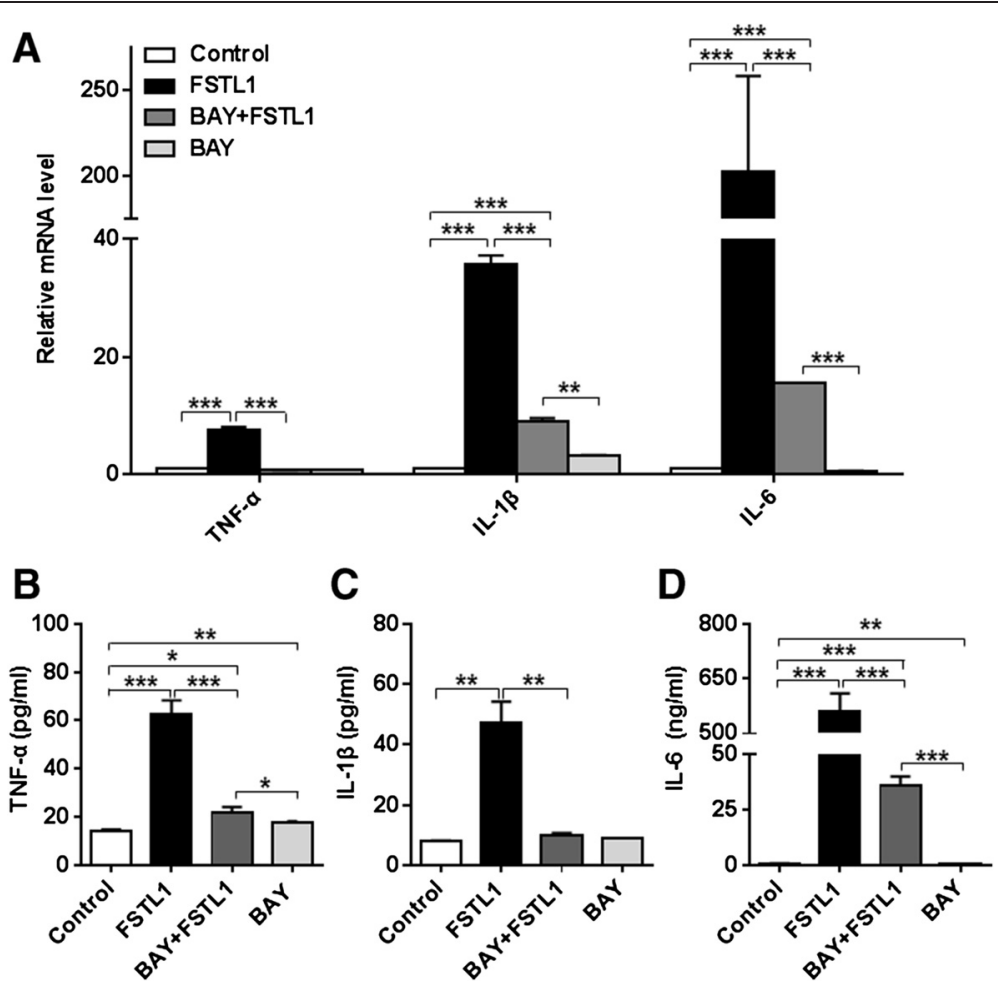

Figure 3 Inhibition of the NF-kB pathway eliminates the proinflammatory effects of FSTL1. (A) FLSs cultured in a 6-well plate were pretreated with BAY 11-7082 for 1 hour before addition of FSTL1, and then incubated with FSTL1 or vehicle (PBS solution) for 48 hrs. The relative levels of TNF- $a, I L-1 \beta$ and IL-6 mRNA were analyzed by real-time PCR as in Figure 1. (B-D) Cell supernatants were collected, and TNF-a, IL-1 $\beta$ and IL-6 concentrations were evaluated by ELISA. All results are presented as mean \pm (standard deviation) SD of three independent experiments (each FLS cell line from an individual patient was used for each experiment). ${ }^{*} P<0.05,{ }^{* *} P<0.01,{ }^{* * *} P<0.001$, relative to the control or between groups as indicated, Student $t$ test. ELISA, enzyme-linked immunosorbent assay; FLS, fibroblast-like synoviocyte; FSTL1, follistatin-like protein 1; IL-1 $\beta$, interleukin-1 $\beta$; IL-6, interleukin-6; NF-KB, nuclear factor kappa B; PBS, phosphate-buffered saline; PCR, polymerase chain reaction; TNF-a, tumor necrosis factor alpha.

FSTL1 contributes to the proinflammatory effects by activating NF-kB signaling in OA FLS.

\section{FSTL1 accelerates the proliferation of FLS in a p53 and p21 dependent way}

Previous work suggests that activation of the NF- $\mathrm{kB}$ signaling pathway is associated with synovial fibroblast hyperplasia, and thus contributes to OA progression [30]. Thus, we wondered if FSTL1 promotes the proliferation of FLS. After FLSs were treated with FSTL1 for 6 consecutive days (FSTL1 was daily added to the medium), an increase of FLS number was significant in the subsequent 5 days (D3 to 7) as indicated in Figure 4A. To further examine if the FSTL1-induced FLS proliferation depends on the expression of two tumor suppressors: p53 and p21, we carried out western blot analysis on D4 and D6. As shown in Figure 4B, FSTL1 significantly inhibited the expression of p53 and p21 on the two time points (the data from D6 not shown). Collectively, these results suggest that FSTL1 enhances the proliferation of FLS in a p53- and p21dependent way in OA FLS.
The elevated level of SF FSTL1 and serum FSTL1 correlates with the increased level of SF IL-6 in OA patients

As shown earlier, FSTL1 significantly improved expression and release of three inflammatory cytokines. In combination with our previous work that both serum and SF levels of FSTL1 are elevated in OA patients [24]. First, we tried to compare the concentrations of these cytokines in serum between OA patients and HC. By ELISA analysis, the three cytokines (TNF- $\alpha$, IL-1 $\beta$ and IL-6) were undetectable in all $\mathrm{HC}$ serum samples. Also, we failed to detect the levels of TNF- $\alpha$ and IL- $1 \beta$ in OA patients. Intriguingly, we observed a marked increase of the SF IL-6 level $(42.08 \pm 73.38 \mathrm{pg} / \mathrm{ml})$ in $58 \mathrm{OA}$ patients. Next, we analyzed the association between FSTL1 (SF and serum) and SF IL-6 in 58 OA patients. As illustrated in Figure 5A-B, the level of SF IL-6 is significantly associated with SF FSTL1 $(r=0.53$, $P<0.0001)$ and also correlated with serum FSTL1 ( $\mathrm{r}=$ $0.34, P=0.0086)$. These data suggest that FSTL1 is involved in osteoarthritis by elevating inflammatory cytokine IL-6. 

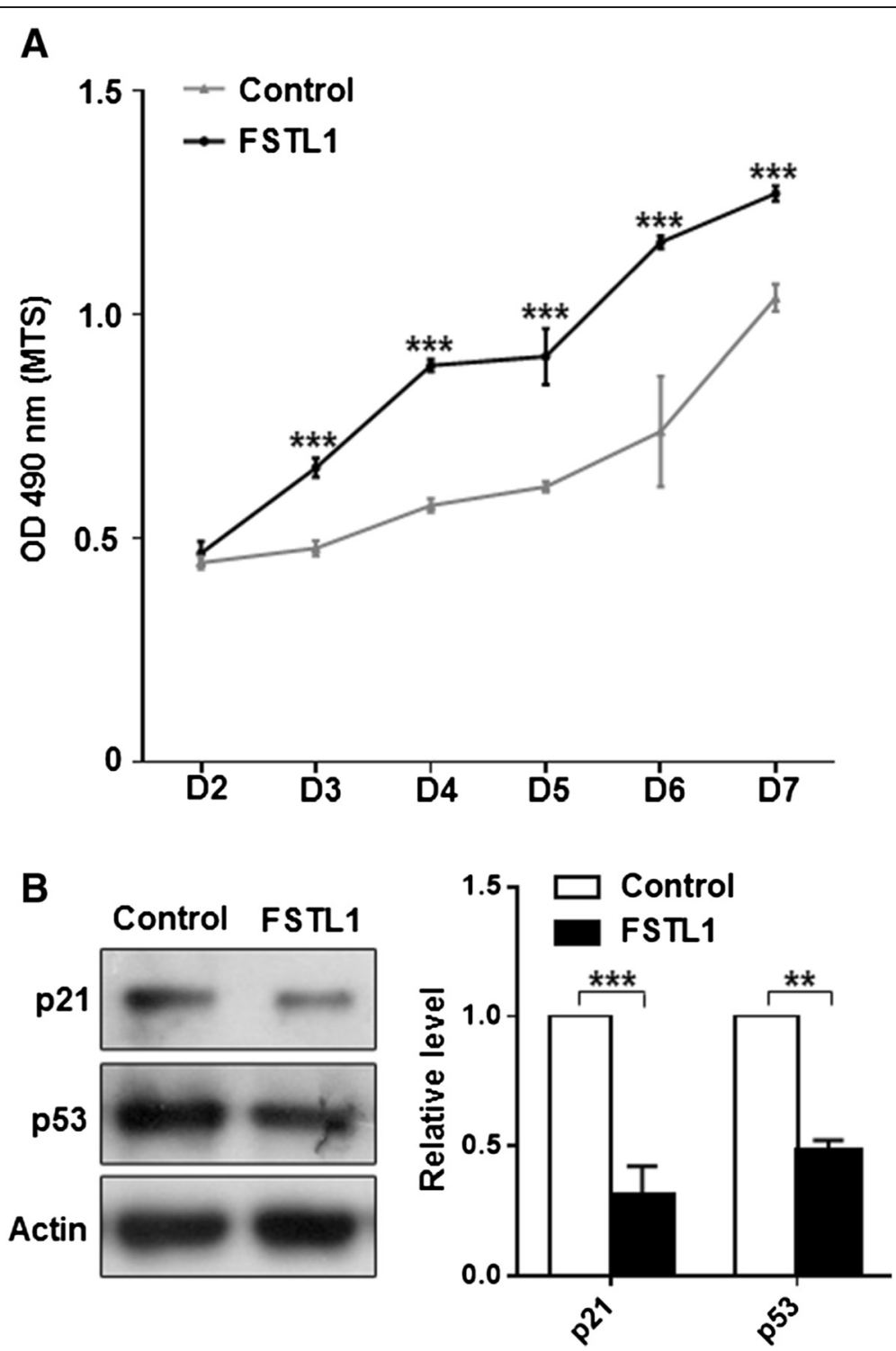

Figure 4 FSTL1 enhances proliferation of FLS in a p53- and p21-dependent way. (A) About 2,000 OA FLS cells per well were seeded into a 96-well plate in triplicate (the seeding day was referred to as Day 0, that is, D0). On the subsequent 6 days (referred as to D1 to D6), $5 \mu \mathrm{g} / \mathrm{ml}$ FSTL1 was daily applied to the medium. MTS test was performed daily on D2 to D7. (B) Cells were collected after D4 exposure to $5 \mu \mathrm{g} / \mathrm{ml}$ FSTL1 and western blots for p53 and p21 were conducted. Left panel, representative blots; Right panel, quantification data. Results in (A) and (B) are presented as mean \pm (standard deviation) SD of three independent experiments (each FLS cell line from an individual patient was used for each experiment). ${ }^{*} P<0.05,{ }^{* * *} P<0.01$, compared to the vehicle-treated control, Student $t$ test. FLS, fibroblast-like synoviocyte; FSTL1, follistatin-like protein 1; MTS, 3-(4,5-dimethylthiazol-2-yl)-5-(3-carboxymethoxyphenyl)-2-(4-sulfophenyl)-2H-tetrazolium, inner salt; OA, osteoarthritis.

\section{Discussion}

As a secreted extracellular glycoprotein, FSTL1 is widely considered to participate in development and immune diseases pathogenesis. And some groups identified FSTL1 as a new proinflammatory mediator that contributes to rheumatoid arthritis by promoting the expression of TNF $\alpha$, IL-1 $\beta$, IL- 6 and IL- 8 , as well as by enhancing the interferon- $\gamma$ (IFN- $\gamma$ ) signaling pathway in a mouse model $[22,31,32]$. In this study, we observed two significant effects of FSTL1 in cultured OA FLS.
One is that FSTL1 remarkably stimulates the expression and secretion of proinflammatory cytokines TNF$\alpha$, IL- $1 \beta$, and especially IL- 6 by activating the NF- $\mathrm{KB}$ signaling pathway. Interestingly, there was an association between elevated FSTL1 levels and significant increased IL- 6 concentrations in SF of OA patients. The other one is that FSTL1 promotes the proliferation of FLS in a p53- and p2-dependent way. These findings further suggest that FSTL1 might be a potential target for the treatment of $\mathrm{OA}$ in humans. 

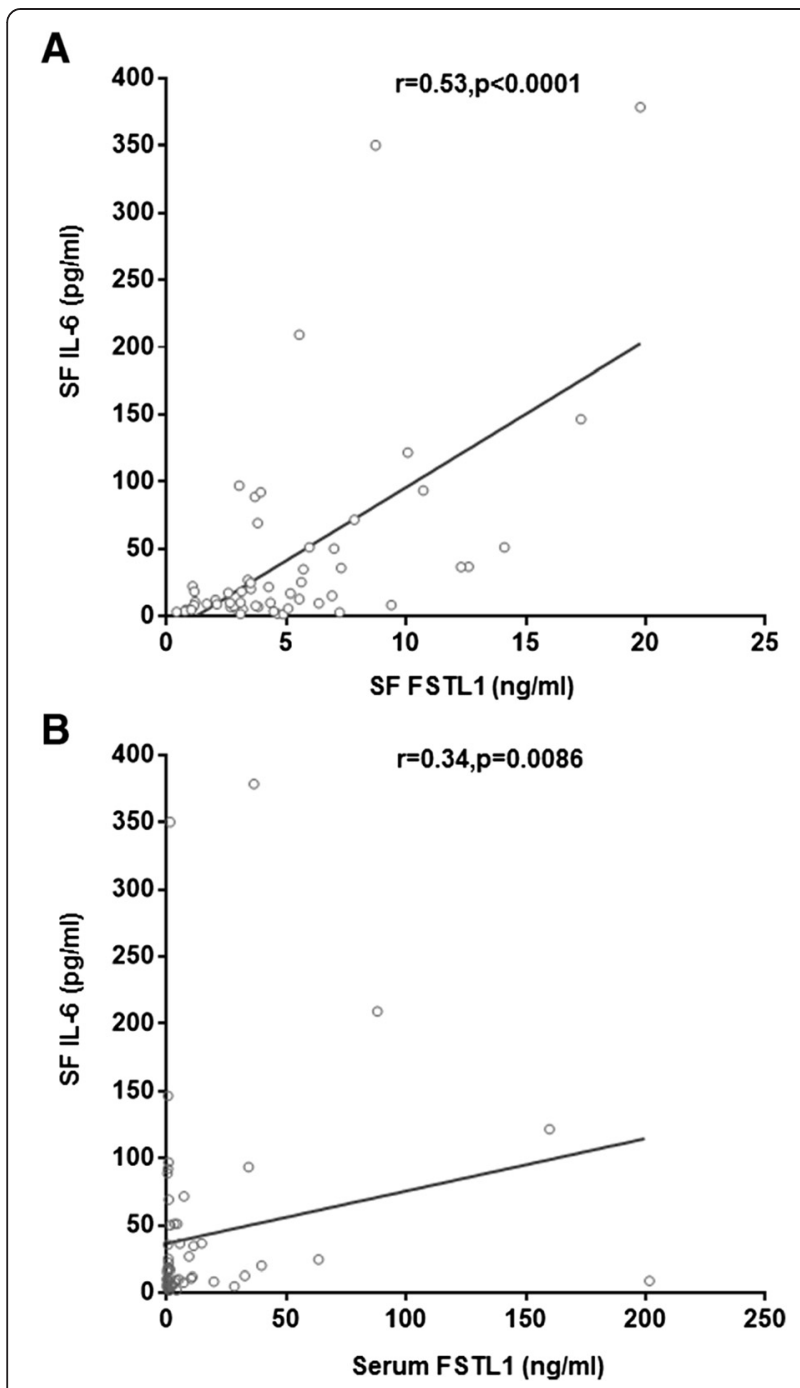

Figure 5 Correlation of SF and serum FSTL1 concentrations with SF IL-6 concentration in OA patients. (A) The association between concentrations of IL-6 and FSTL1 in SF of OA patients was evaluated. (B) The association between concentrations of SF IL-6 and serum FSTL1 in OA patients was evaluated. Fifty-eight paired samples (serum versus SF) were collected from 58 OA patients. Correlation coefficients $(r)$ and $P$ values from the Spearman rank-order test are shown. Each point represents an individual value. FSTL1, follistatin-like protein 1; IL-6, interleukin-6; OA, osteoarthritis; SF, synovial fluid.

TNF- $\alpha$, IL- $1 \beta$ and IL- 6 are thought to be the key proinflammatory cytokines related to the pathophysiology of OA [33]. A number of studies revealed that the function of TNF- $\alpha$ is involved in activation of the inflammatory cascades and IL- $1 \beta$ is a major player of cartilage erosion in OA [34-37]. IL-6 is also reported to exert a proinflammatory role in degenerative joint disease [37]. Importantly, the serum and SF levels of IL- 6 are elevated in OA patients [38,39], consistent with our results. Moreover, IL-6 attributes to subchondral bone damage in $\mathrm{OA}$ by triggering osteoclast differentiation and bone resorption [40]. Our prior work showed that the level of increased serum FSTL1 may be identified as a potential serum marker related to the severity of joint damage of OA [23]. Taken together, FSTL1-induced IL-6 overexpression plays a key role in exaggerating OA progression. In this study, we further established the correlation between serum FSTL1 level and IL-6 concentrations in SF. FSTL1 exerts its proinflammatory role by changing the microenvironment of synoviocytes and directly stimulating secretion of inflammatory cytokines by FLS.

To further understand the role of FSTL1 in OA progression, we aimed to search for its receptor on FLS membrane. Murakami and his coworkers reported that a disco-interacting protein 2 homolog a (DIP2a) from Drosophila was a candidate receptor for FSTL1 in endothelial cells [39]. It exerted the anti-apoptotic and promigratory effects of FSTL1 and mediated FSTL1induced activation of Akt $[41,42]$. But so far there are no reports of the existence of DIP2a in synovium or FLSs. In our primary FLS culture, only a few of DIP2apositive cells were detected by immunohistochemistry staining. Furthermore, we knocked down of DIP2a with small interfering RNA (siRNA) (the effectiveness of knockdown was confirmed in HEK293T cells), but failed to observe statistically significant alterations in FSTL1-induced inflammatory cytokines. Therefore, it seems that the effects of FSTL1 may not be mediated by DIP2a in OA FLS. There is evidence that FSTL1 can bind to CD14 and elicits an innate immune response via Toll-like receptor 4 (TLR4) [43]. In HEK293T, FSTL1 activates NF- $\mathrm{kB}$ and elevates the level of IL-6 and IL-8 [43]. Thus it is likely that in OA FLS, FSTL1 promotes inflammation via the CD14-TLR4 pathway rather than DIP2a. In addition, we cannot exclude that the effects of FSTL1 is achieved via the TGF superfamily, since FSTL1 interacts with the TGF- $\beta$ superfamily, including activin, TGF- $\beta$, bone morphogenetic protein (BMP)-2/4, and their receptors $[42,44]$.

Our results show that FSTL1 activates the NF- $\mathrm{kB}$ pathway upon binding to its unidentified receptor in OA FLS. We provide evidence that the active transcriptional element p65 is recruited to the promoter region of TNF- $\alpha$ and IL-6, and as a result directly enhances the expression level of these cytokines. Since TNF- $\alpha$ and IL- $1 \beta$ also can be induced by activation of p38 and c-Jun N-terminal kinase (JNK) and their downstream transcriptional factor AP1 [45], it may be an explanation of the not fully abolished level of the cytokines after inhibition of NF-kB signaling by BAY 11-7082. Furthermore, it was reported that FSTL1 contributes to arthritis as a proinflammatory mediator by enhancing the IFN- $\gamma$ signaling pathway in a collagen-induced arthritis (CIA) mouse model [32]. Overall, FSTL1 can activate multi-proinflammatory pathways to participate in OA pathophysiology. 
In this study, we also observed that FSTL1 enhances FLSs proliferation, suggesting that FSTL1 acts as a synoviopathy-inducing factor to be involved in OA pathogenesis by the increasing expansiveness of FLS. It is thought that a pannus formed by abnormal proliferative FLS is a hallmark of rheumatoid arthritis and OA, finally damaging articular bone and cartilage [46]. Since p53, a tumor-suppressor protein, plays a pivotal role in cell cycle regulation and programmed cell death, we intended to determine whether the overgrowth is due to the inhibition of its activity in the presence of FSTL1. Our western blot analysis supported the idea. Supportably, p53 serves as an intracellular anti-inflammatory function [47]. The absence of p53 is involved in both decreased apoptosis of FLS and increased synovial IL-6 production in a CIA mouse model [48]. p21, a cyclin-dependent kinase inhibitor, is induced by $\mathrm{p} 53$, and participates in the pathogenesis of OA by blocking of overexpressed IL-6 and matrix metalloproteinases (MMPs) synthesis [49]. Consistently, we also observed that the application of FSTL1 markedly downregulated the expression of p21, implicating the involvement of p21 in the proliferative effects of FSTL1.Therefore, we propose that FSTL1 exacerbates OA by facilitating expansiveness of FLS as a synoviopathy-inducing factor besides increasing secretion of inflammatory cytokines. Like many inflammatory factors (such as interleukin-17 (IL-17)) [50], FSTL1 has 'dual power' to exacerbate arthritis.

\section{Conclusions}

FSTL1 significantly promotes the expression of inflammatory cytokines (TNF- $\alpha$, IL-1 $\beta$ and IL-6) in cultured FLS from OA patients, which is achieved by activating the NF- $\kappa B$ pathway. Moreover, FSTL1 improves FLS proliferation in a p53- and p21-dependent pathway. Finally, the elevated concentration of SF IL- 6 is correlated with serum and SF FSTL1 levels in OA patients. These findings suggest that FSTL1 is involved in the progression of synovial inflammation and hyperplasia in OA, and presumably is a potential target for OA therapy.

\footnotetext{
Abbreviations

BMP: bone morphogenetic protein; ChIP: chromatin immunoprecipitation; CIA: collagen-induced arthritis; DIP2a: disco-interacting protein 2 homolog a; DMEM: Dulbecco's modified Eagle's medium; DMSO: dimethyl sulphoxide; ECM: extracellular matrix; ELISA: enzyme-linked immunosorbent assay; FBS: fetal bovine serum; FLS: fibroblast-like synoviocyte; FSTL1: follistatin-like protein 1; GAPDH: glyceraldehyde 3-phosphate dehydrogenase; HC: healthy control; IgG: immunoglobulin G; IKB: inhibitor of kappa B; IKK: IKB kinases; IFN- $\gamma$ : interferon- $\gamma$; IL-1ß: interleukin-1ß; IL-6: interleukin-6; IL-8: interleukin-8; IL-17: interleukin-17; JNK: c-Jun N-terminal kinase; KL: Kellgren-Lawrence; MMP: matrix metalloproteinase; MTS: 3-(4,5-dimethylthiazol-2-yl)-5-(3-carboxymethoxyphenyl)2-(4-sulfophenyl)-2H-tetrazolium, inner salt; NF-kB: nuclear factor kappa B; OA: osteoarthritis; PBS: phosphate-buffered saline; PCR: polymerase chain reaction; PES: phenazine ethosulfate; PVDF: polyvinylidene fluoride; SF: synovial fluid; SDS: sodium dodecyl sulfate; siRNA: small interfering RNA; TGF- $\beta 1$ : transforming factor $\beta 1$; TLR4: Toll-like receptor 4; TNF-a: tumor necrosis factor alpha.
}

\section{Competing interests}

The authors declare that they have no competing interests.

\section{Authors' contributions}

YW contributed to the conception and design of the study. SN and KM carried out the principal experiments. KM, CL, RS, NX, and RZ completed the acquisition or preparation of clinical samples. SN, KM, XZ, NX, CL, RZ, RS and YW contributed to the analysis and interpretation of the data. $S N, K M, X Z, C L$, $R S, R Z$ and $Y W$ contributed to the drafting of the manuscript. SN, KM, YW, XZ and NX contributed to the critical revision for important intellectual content. All authors read and approved the final manuscript.

\section{Acknowledgements}

We thank Dr. Dawei Li (College of Physicians and Surgeons, Columbia University) for valuable suggestions and critical comments on our manuscript. This work was supported by grants from the National Natural Science Foundation of China (81171680 to Y.W.), the science and technology support of Changzhou (CE20135054 to Y.W.), and the Key Project of Changzhou Health Bureau (ZD201003 to Y.W.).

\section{Author details}

'Department of Orthopedics, The Affiliated Hospital of Nanjing Medical University, Changzhou No.2 People's Hospital, 29 Xinglong Alley, Changzhou 213003, China. 'Laboratory of Clinical Orthopedics, The Affiliated Hospital of Nanjing Medical University, Changzhou No.2 People's Hospital, 29 Xinglong Alley, Changzhou 213003, China. ${ }^{3}$ Department of Neurology, Laboratory of Neurological Diseases, The Affiliated Hospital of Nanjing Medical University, Changzhou No.2 People's Hospital, 29 Xinglong Alley, Changzhou 213003, China.

Received: 28 October 2014 Accepted: 23 March 2015

Published online: 02 April 2015

\section{References}

1. Hunter DJ, Nevitt M, Losina E, Kraus V. Biomarkers for osteoarthritis: Current position and steps towards further validation. Best Pract Res Clin Rheumatol. 2014;28:61-71.

2. de Lange-Brokaar BJ, loan-Facsinay A, van Osch GJ, Zuurmond AM, Schoones J, Toes RE, et al. Synovial inflammation, immune cells and their cytokines in osteoarthritis: a review. Osteoarthritis Cartilage. 2012;20:1484-99.

3. Buckwalter JA, Mankin HJ, Grodzinsky AJ. Articular cartilage and osteoarthritis. Instr Course Lect. 2005;54:465-80.

4. Pelletier JP, Martel-Pelletier J, Abramson SB. Osteoarthritis, an inflammatory disease: potential implication for the selection of new therapeutic targets. Arthritis Rheum. 2001:44:1237-47.

5. Smith MD, Triantafillou S, Parker A, Youssef PP, Coleman M. Synovial membrane inflammation and cytokine production in patients with early osteoarthritis. J Rheumatol. 1997;24:365-71.

6. Steenvoorden MM, Bank RA, Ronday HK, Toes RE, Huizinga TW, DeGroot J. Fibroblast-like synoviocyte-chondrocyte interaction in cartilage degradation. Clin Exp Rheumatol. 2007;25:239-45.

7. Fuchs S, Skwara A, Bloch M, Dankbar B. Differential induction and regulation of matrix metalloproteinases in osteoarthritic tissue and fluid synovial fibroblasts. Osteoarthritis Cartilage. 2004;12:409-18.

8. Nair A, Kanda V, Bush-Joseph C, Verma N, Chubinskaya S, Mikecz K, et al. Synovial fluid from patients with early osteoarthritis modulates fibroblast-like synoviocyte responses to toll-like receptor 4 and toll-like receptor 2 ligands via soluble CD14. Arthritis Rheum. 2012;64:2268-77.

9. Sun Y, Firestein GS, Wenger L, Huang CY, Cheung HS. Telomerasetransduced osteoarthritic fibroblast-like synoviocyte cell line. Biochem Biophys Res Commun. 2004;323:1287-92.

10. Marcu KB, Otero M, Olivotto E, Borzi RM, Goldring MB. NF-kappaB signaling: multiple angles to target OA. Curr Drug Targets. 2010;11:599-613.

11. Scanzello CR, Goldring SR. The role of synovitis in osteoarthritis pathogenesis. Bone. 2012;51:249-57.

12. Cogswell PC, Scheinman Rl, Baldwin Jr AS. Promoter of the human NFkappa B p50/p105 gene. Regulation by NF-kappa B subunits and by C-REL. J Immunol. 1993;150:2794-804.

13. Siebenlist U, Franzoso G, Brown K. Structure, regulation and function of NF-kappa B. Annu Rev Cell Biol. 1994;10:405-55. 
14. Hacker H, Karin M. Regulation and function of IKK and IKK-related kinases. Sci STKE. 2006; 2006:re13.

15. Bonizzi G, Karin M. The two NF-kappaB activation pathways and their role in innate and adaptive immunity. Trends Immunol. 2004:25:280-8.

16. Li Y, Ahmed F, Ali S, Philip PA, Kucuk O, Sarkar FH. Inactivation of nuclear factor kappaB by soy isoflavone genistein contributes to increased apoptosis induced by chemotherapeutic agents in human cancer cells. Cancer Res. 2005;65:6934-42.

17. Baldwin Jr AS. The NF-kappa B and I kappa B proteins: new discoveries and insights. Annu Rev Immunol. 1996;14:649-83.

18. Shibanuma M, Mashimo J, Mita A, Kuroki T, Nose K. Cloning from a mouse osteoblastic cell line of a set of transforming-growth-factor-beta 1-regulated genes, one of which seems to encode a follistatin-related polypeptide. Eur J Biochem. 1993;217:13-9.

19. Sundaram GM, Common JE, Gopal FE, Srikanta S, Lakshman K, Lunny DP, et al. 'See-saw' expression of microRNA-198 and FSTL1 from a single transcript in wound healing. Nature. 2013;495:103-6.

20. Gorgens SW, Raschke S, Holven KB, Jensen J, Eckardt K, Eckel J. Regulation of follistatin-like protein 1 expression and secretion in primary human skeletal muscle cells. Arch Physiol Biochem. 2013;119:75-80.

21. Shimano M, Ouchi N, Nakamura K, van Wijk B, Ohashi K, Asaumi Y, et al. Cardiac myocyte follistatin-like 1 functions to attenuate hypertrophy following pressure overload. Proc Natl Acad Sci U S A. 2011;108:E899-906.

22. Chaly Y, Marinov AD, Oxburgh L, Bushnell DS, Hirsch R. FSTL1 promotes arthritis in mice by enhancing inflammatory cytokine/chemokine expression. Arthritis Rheum. 2012;64:1082-8.

23. Li D, Wang Y, Xu N, Wei Q, Wu M, Li X, et al. Follistatin-like protein 1 is elevated in systemic autoimmune diseases and correlated with disease activity in patients with rheumatoid arthritis. Arthritis Res Ther. 2011;13:R17

24. Wang Y, Li D, Xu N, Tao W, Zhu R, Sun R, et al. Follistatin-like protein 1: a serum biochemical marker reflecting the severity of joint damage in patients with osteoarthritis. Arthritis Res Ther. 2011;13:R193.

25. Kellgren JH, Lawrence JS. Radiological assessment of osteo-arthrosis. Ann Rheum Dis. 1957:16:494-502

26. Altman R, Asch E, Bloch D, Bole G, Borenstein D, Brandt $K$, et al. Development of criteria for the classification and reporting of osteoarthritis. Classification of osteoarthritis of the knee Diagnostic and Therapeutic Criteria Committee of the American Rheumatism Association. Arthritis Rheum. 1986;29:1039-49.

27. Frank S, Peters MA, Wehmeyer C, Strietholt S, Koers-Wunrau C, Bertrand J, et al. Regulation of matrixmetalloproteinase- 3 and matrixmetalloproteinase- 13 by SUMO-2/3 through the transcription factor NF-kappaB. Ann Rheum Dis. 2013;72:1874-81.

28. Pahl HL. Activators and target genes of Rel/NF-kappaB transcription factors. Oncogene. 1999;18:6853-66.

29. Hayden MS, Ghosh S. Signaling to NF-kappaB. Genes Dev. 2004;18:2195-224.

30. Franke S, Sommer M, Ruster C, Bondeva T, Marticke J, Hofmann G, et al. Advanced glycation end products induce cell cycle arrest and proinflammatory changes in osteoarthritic fibroblast-like synovial cells. Arthritis Res Ther. 2009;11:R136.

31. Kawabata D, Tanaka M, Fujii T, Umehara H, Fujita Y, Yoshifuji H, et al. Ameliorative effects of follistatin-related protein/TSC-36/FSTL1 on joint inflammation in a mouse model of arthritis. Arthritis Rheum. 2004:50:660-8.

32. Clutter SD, Wilson DC, Marinov AD, Hirsch R. Follistatin-like protein 1 promotes arthritis by up-regulating IFN-gamma. J Immunol. 2009;182:234-9.

33. Kapoor M, Martel-Pelletier J, Lajeunesse D, Pelletier JP, Fahmi H. Role of proinflammatory cytokines in the pathophysiology of osteoarthritis. Nat Rev Rheumatol. 2011;7:33-42

34. Chadjichristos C, Ghayor C, Kypriotou M, Martin G, Renard E, Ala-Kokko L, et al. Sp1 and Sp3 transcription factors mediate interleukin-1 beta down-regulation of human type II collagen gene expression in articular chondrocytes. J Biol Chem. 2003:278:39762-72.

35. Shakibaei M, Schulze-Tanzil G, John T, Mobasheri A. Curcumin protects human chondrocytes from IL-|1 beta-induced inhibition of collagen type II and beta1-integrin expression and activation of caspase-3: an immunomorphological study. Ann Anat. 2005;187:487-97.

36. Guerne PA, Carson DA, Lotz M. IL-6 production by human articular chondrocytes. Modulation of its synthesis by cytokines, growth factors, and hormones in vitro. J Immunol. 1990;144:499-505
37. Lotz M, Terkeltaub R, Villiger PM. Cartilage and joint inflammation. Regulation of IL-8 expression by human articular chondrocytes. J Immunol. 1992;148:466-73.

38. Kaneko S, Satoh T, Chiba J, Ju C, Inoue K, Kagawa J. Interleukin-6 and interleukin-8 levels in serum and synovial fluid of patients with osteoarthritis. Cytokines Cell Mol Ther. 2000:6:71-9.

39. Uson J, Balsa A, Pascual-Salcedo D, Cabezas JA, Gonzalez-Tarrio JM, Martin-Mola E, et al. Soluble interleukin 6 (IL-6) receptor and IL-6 levels in serum and synovial fluid of patients with different arthropathies. J Rheumatol. 1997;24:2069-75.

40. Kwan Tat S, Padrines M, Theoleyre S, Heymann D, Fortun Y. IL-6, RANKL, TNF-alpha/L-1: interrelations in bone resorption pathophysiology. Cytokine Growth Factor Rev. 2004;15:49-60.

41. Ouchi N, Asaumi Y, Ohashi K, Higuchi A, Sono-Romanelli S, Oshima Y, et al. DIP2A functions as a FSTL1 receptor. J Biol Chem. 2010;285:7127-34.

42. Tanaka M, Murakami K, Ozaki S, Imura Y, Tong XP, Watanabe T, et al. DIP2 disco-interacting protein 2 homolog A (Drosophila) is a candidate receptor for follistatin-related protein/follistatin-like 1-analysis of their binding with TGF-beta superfamily proteins. FEBS J. 2010;277:4278-89.

43. Murakami K, Tanaka M, Usui T, Kawabata D, Shiomi A, Iguchi-Hashimoto M, et al. Follistatin-related protein/follistatin-like 1 evokes an innate immune response via CD14 and toll-like receptor 4. FEBS Lett. 2012;586:319-24.

44. Zhou J, Liao M, Hatta T, Tanaka M, Xuan X, Fujisaki K. Identification of a follistatin-related protein from the tick Haemaphysalis longicornis and its effect on tick oviposition. Gene. 2006;372:191-8.

45. Wang C, Deng L, Hong M, Akkaraju GR, Inoue J, Chen ZJ. TAK1 is a ubiquitin-dependent kinase of MKK and IKK. Nature. 2001;412:346-51.

46. Ota F, Maeshima A, Yamashita S, Ikeuchi H, Kaneko Y, Kuroiwa T, et al. Activin A induces cell proliferation of fibroblast-like synoviocytes in rheumatoid arthritis. Arthritis Rheum. 2003:48:2442-9.

47. Sweeney SE, Firestein GS. Signal transduction in rheumatoid arthritis. Curr Opin Rheumatol. 2004;16:231-7.

48. Yao Q, Wang S, Glorioso JC, Evans CH, Robbins PD, Ghivizzani SC, et al. Gene transfer of p53 to arthritic joints stimulates synovial apoptosis and inhibits inflammation. Mol Ther. 2001;3:901-10.

49. Perlman H, Bradley K, Liu H, Cole S, Shamiyeh E, Smith RC, et al. IL-6 and matrix metalloproteinase-1 are regulated by the cyclin-dependent kinase inhibitor p21 in synovial fibroblasts. J Immunol. 2003;170:838-45.

50. Lee SY, Kwok SK, Son HJ, Ryu JG, Kim EK, Oh HJ, et al. IL-17-mediated BCl-2 expression regulates survival of fibroblast-like synoviocytes in rheumatoid arthritis through STAT3 activation. Arthritis Res Ther. 2013;15:R31.

\section{Submit your next manuscript to BioMed Central and take full advantage of:}

- Convenient online submission

- Thorough peer review

- No space constraints or color figure charges

- Immediate publication on acceptance

- Inclusion in PubMed, CAS, Scopus and Google Scholar

- Research which is freely available for redistribution 